\title{
Kernos
}

Revue internationale et pluridisciplinaire de religion grecque antique

9 | 1996

Varia

\section{Que l'on ne doit pas blâmer les cités sans gardiens ou mal gardées}

Le serment des rois atlantes (Critias, 119c-120c)

Marie-Laurence Desclos

\section{(2) OpenEdition}

Journals

Édition électronique

URL : http://journals.openedition.org/kernos/1181

DOI : 10.4000/kernos. 1181

ISSN : 2034-7871

Éditeur

Centre international d'étude de la religion grecque antique

Édition imprimée

Date de publication : 1 janvier 1996

ISSN : 0776-3824

Référence électronique

Marie-Laurence Desclos, «Que l'on ne doit pas blâmer les cités sans gardiens ou mal gardées ", Kernos [En ligne], 9 | 1996, mis en ligne le 21 avril 2011, consulté le 30 avril 2019. URL : http:// journals.openedition.org/kernos/1181; DOI : 10.4000/kernos.1181 


\title{
Que l'on ne doit pas blâmer les cités sans gardiens ou mal gardées.
}

\author{
Le serment des rois atlantes (Critias, 119c-120c)
}

\begin{abstract}
Si quelqu'un approuvait l'élevage des chèvres et disait de l'animal lui-même que c'est une richesse appréciable, mais qu'un autre, pour avoir vu des chèvres paître sans chevrier dans des terres cultivées et y commettre des dégâts, se mît à les critiquer, ou qu'il blâmât de même toutes les bêtes qu'il aurait vues sans gardiens ou mal gardées, croyons-nous qu'une pareille critique eût tant soit peu de bon sens?
\end{abstract}

Lois, I, 639a2-7

Toute une série de travaux a, ces dernières années, considérablement enrichi la bibliographie platonicienne concernant le Timée (17a-27b) et le Critias $^{1}$. On doit cependant constater qu'il n'en est pas de même pour ce texte étrange qui décrit le rituel auquel les rois atlantes, s'il faut en croire Platon, se livraient périodiquement. Il convient d'en rappeler rapidement la teneur.

Une fois tous les cinq ou six ans, le pouvoir est mis en commun et les rois peuvent exercer leur autorité les uns sur les autres ${ }^{2}$. "Ils étaient jugés et ils jugeaient $\left(120_{\mathrm{c} 2}\right)^{3}$. Mais, pour ce faire, ils doivent s'inspirer mutuellement confiance $e^{4}$ par un cérémonial que l'on a dès longtemps identifié à une «ordalie

1 Il suffira, pour s'en convaincre, de se reporter à la bibliographie du Timée (17a-27b) et du Critias, dans les quatre derniers numéros de la revue Lustrum consacrés à Platon : 20 (1977), 25 (1983), 30 (1988) et 34 (1992). Sauf indication contraire, je suivrai la traduction de L. BRISSON, Platon - Timée / Crittas, Paris, 1992.

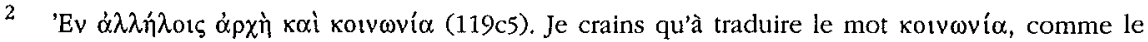
fait A. Rivaud dans la Collection des Universités de France, par « rapports» des rois entre eux, ou même, comme L. Brisson, par « relations mutuelles », on passe à côté du sens véritable de ce passage. Une fois tous les cinq ou six ans, les décrets de Poséidon permettent de corriger les effets de la division de l'île en dix portions, et de son corollaire, la division du pouvoir. Ces parties sont parties d'un tout qu'il convient périodiquement de reformer. Or quel meilleur endroit pour ce faire

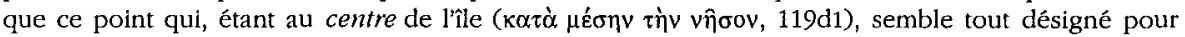
la mise en commun? Sur la synonymie politique des expressions \&́v kolvậ et év $\mu \varepsilon \sigma \hat{a}, c f$. J.-P. VERNANT, Mythe et pensée chez les Grecs, Paris, 1974, I, p. 177-180.

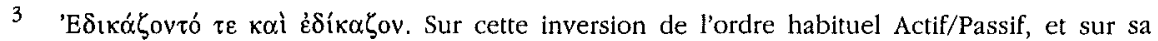
signification, cf. H. HERTER, Das Königsritual der Atlantis, in RhM, 109 (1966), p. 257-258.

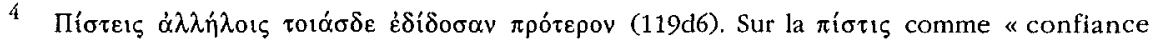
inspirée à d'autres ou que d'autres inspirent », $c f$. P. CHANTRAINE, Dictionnatre étymologique de la langue grecque. Histotre des mots, Paris, 1968, s.v. $\pi \varepsilon i \theta 0 \mu \alpha 1$, p. 869a. 
par voie de communion $»^{5}$. Quels en sont les différents moments ? Après s'être procuré une victime sacrificielle parmi les taureaux courant dans le téménos de Poséidon, ils la conduisent vers une colonne d'orichalque où ils l'égorgent. Détail ayant son importance : sur cette colonne sont gravées les lois données par le dieu, ainsi qu'un serment proférant de terribles imprécations contre qui les violerait. Et voici le passage qui m'intéresse tout particulièrement :

Lors donc que, après avoir effectué le sacrifice conformément à leurs lois, ils brûlaient tous les membres du taureau, après avoir rempli de vin trempé un cratère, ils jetaient au nom de chacun d'eux un caillot du sang dans ce cratère; après avoir nettoyé la colonne sur toute sa surface, ils le mettaient au feu. Puis, puisant avec des coupes d'or dans le cratère et versant des libations dans le feu, ils faisaient le serment de rendre la justice en fonction des lois inscrites sur la stèle, de châtier celui d'entre eux qui, antérieurement, y aurait contrevenu sur quelque point, de ne contrevenir de leur plein gré à l'avenir sur aucun point aux ordres de la prescription, de ne commander et de n'obéir que conformément aux lois de leur père. Après avoir pris cet engagement pour lui-même en particulier et pour sa descendance, chaque roi buvait et remettait la coupe en ex-voto dans le sanctuaire du dieu (119e-120b4).

Ce n'est qu'à la nuit venue que, revêtant des robes bleu nuit, ils s'asseyaient par terre dans les cendres du sacrifice, et jugeaient.

Outre son exotisme un peu inquiétant, le rituel sacrificiel qui nous est ici décrit a pour intérêt d'entretenir un rapport direct et explicite avec l'exercice du pouvoir et sa conformité aux règles édictées par la divinité. Reste à savoir quelle peut être sa fonction dans l'économie du double récit de Critias, dont on a montré qu'il ne prenait sens que par sa mise en parallèle avec la situation politique de l'Athènes historique telle qu'elle était conçue par Platon ${ }^{6}$. Pour tenter de répondre à cette question, je ferai plusieurs constats.

5 Cf., par exemple, A. VINCENT, Essai sur le sacrifice de communion des rois atlantes dans le « Critias» de Platon (119c5-120c4), in Mémorlal Lagrange, Paris, 1940, p. 92. Voir également Ch. PICARD, Une source possible de Platon pour le "Jugement des rois " de l'Atlantide, in L'Acropole, 8 (1933), p. 8-9; A. RIVAUD, Notice du Critias, Paris, $1970^{5}$ [Paris, 1925], p. 245, ainsi que l'examen critique qu'en fait HERTER, art. cht. (n. 3), p. 251. L. BRISSON, De la philosophie politique à l'épopée. Le "Critlas" de Platon, in RMM, 75 (1970), p. 433, ne remet pas en question cette lecture.

6 Cf. P. VIDAL-NAQUET, Athènes et l'Atlantide. Structure et signification d'un mythe platonicien, in Le chasseur noir. Formes de pensée et formes de société dans le monde grec, Paris, 1981, p. 335-360; Hérodote et l'Atlantide: entre les Grecs et les Juifs. Réflextons sur l'bistortographie du siècle des Lumières, in QS, 16 (1982), p. 5-75, et surtout les pages 44-46. 
1. Le Timée comme le Critias, ainsi que l'Hermocrate qui n'a jamais été écrit, sont présentés par Socrate comme la suite de l'entretien tenu la veille sur «l'organisation de la cité », entretien qui visait à établir « quelle était (...) la constitution la meilleure et quelle sorte d'hommes elle exigeait » (Tim., 17c13). Il s'agit, on le sait, de la République ${ }^{7}$.

2. Cette « suite » a pour but avoué de soumettre la cité idéale à l'épreuve

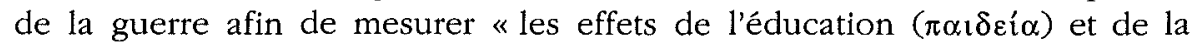
formation ( $\left.\tau \rho \circ \eta)^{\prime}\right)$ données aux citoyens » (19c5-9).

3. Aussi bien est-ce pour cela que l'on choisira les orateurs susceptibles de remplir cette tâche en fonction, justement, de l'éducation qu'ils ont reçue (19e1, 20a1,8).

Je formulerai donc l'hypothèse suivante: notre texte illustre à sa manière la nécessité d'une $\pi \alpha 1 \delta \varepsilon$ í $\alpha$ des gouvernants, seule capable, à long terme, de préserver autant que faire se peut la rectitude de toute constitution. Sans cette $\pi \alpha 1 \delta \varepsilon i \alpha$, et quelle que soit l'origine d'une telle constitution, les garanties dont elle s'entoure et le bon naturel de ceux qui la servent, rien ne pourra s'opposer à une destruction devenue inévitable.

\section{Les dix rois atlantes et la dodécarchie égyptienne}

Je prendrai pour point de départ de cette analyse un rapprochement que l'on a cru pouvoir établir entre les libations des rois atlantes et tel passage des Histoires d'Hérodote (II, 147, 151). Il y est question d'une dodécarchie dont les douze membres se livrent à un rituel, incluant sacrifice et libations, afin de renforcer leur union.

Hans Herter $^{8}$ est, à ma connaissance, le premier qui ait renvoyé au texte d'Hérodote. Il le fait dans une très courte note où il affirme que c'est à une même souveraineté collective que les douze rois égyptiens et les dix rois atlantes sont mutuellement assujettis. Est-ce pour éclairer le sens de l'expres-

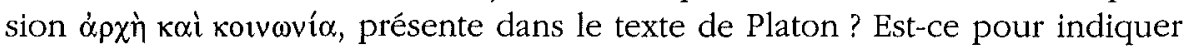
que l'existence historique d'une telle " souveraineté collective » est bien attestée, et n'est pas en soi une invention de Platon? Ou bien considère-t-il que Platon pensait - mais pour quelle raison? - à ce passage du livre II des Histoires en décrivant le rituel sacrificiel du Critias? Nous ne le saurons pas.

Christopher Gill ${ }^{9}$ est un peu plus loquace. D'après lui, la référence s'impose à cet épisode de l'histoire de l'ancienne Égypte raconté par Hérodote. Surtout si l'on veut bien se souvenir que, comme les rois atlantes, les douze rois égyptiens créèrent, eux aussi, un complexe technique et architectural étonnant.

\footnotetext{
7 Pour un état de la question concernant les rapports du Timée et de la République, cf. l'«Introduction » au Critias dans l'édition de L. BRISSON, p. 333-341.

8 HERTER, art. cit. (n. 3), p. 237 et n. 7.

9 Ch. GILL, Plato: the Atlantis story. "Timaeus » 17-27, "Critlas", with Introduction, Notes and Vocabulary, Bristol, 1980, p. 69, note à Critlas $119 \mathrm{~d} 7$.
} 
En témoignent les chapitres 148 à 150. Enfin, il formule l'hypothèse selon laquelle la destruction de cette dodécarchie par la prise du pouvoir d'un seul de ses membres pourrait avoir constitué la façon dont Platon entendait continuer son récit inachevé.

John Gwin Griffiths ${ }^{10}$, quant à lui, voit dans notre passage l'une des multiples traces, dans le double récit de Critias, d'une tradition gréco-égyptienne dont Platon se serait inspiré. Son but étant ici de décrire une situation politique semblable à celle qui précède l'accession de Psammétique au pouvoir, c'est tout naturellement qu'Hérodote lui aurait servi de modèle. La chose est d'autant moins étonnante que, d'entrée de jeu (Tim., 21c), le récit de Critias est placé sous le signe du voyage égyptien de Solon.

Certes, ces trois auteurs ont le mérite de souligner l'importance des chapitres 147 et 151 du livre II des Histoires pour la compréhension du rituel sacrificiel décrit par Platon. Cependant, soit cette importance n'est pas véritablement justifiée : c'est le cas de $\mathrm{H}$. Herter et, dans une moindre mesure, de Ch. Gill; soit la justification est si générale qu'elle pourrait s'appliquer à bien d'autres passages du Critias: je pense, bien sûr, aux explications de J.G. Griffiths. Il m'a donc semblé que le travail n'était pas achevé, et qu'il convenait d'ouvrir le dossier à nouveaux frais. Ma tâche consistera donc tout d'abord à rendre raison de l'élection de ce moment précis du rituel - les libations effectuées par les rois Atlantes - de préférence à tout autre. Puis, étant entendu que le renvoi à Hérodote doit être compris comme une allusion à « une situation politique donnée »- sur ce point il n'y a aucune divergence entre H. Herter, Ch. Gill et J.G. Griffiths -, il m'appartiendra de m'interroger sur la nature de cette situation politique. S'il est vrai qu'il s'agit d'une situation de guerre civile que l'on retrouve, plus ou moins latente, d'un bout à l'autre du récit de Critias, est-elle le résultat d'une quelconque «faute originelle» des Atlantes ? Plusieurs éléments semblent militer en faveur de cette interprétation : le mélange, originel justement, du divin et de l'humain dans le $\gamma^{\prime} \dot{v}$ os atlante ${ }^{11}$; le fait que l'Atlantide est sous le signe du Deux, lequel, dans l'imaginaire grec, dit la

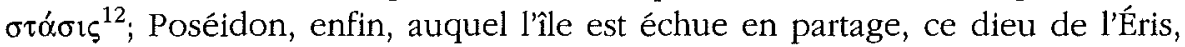
mais d'une ěpı dont l'issue est toujours malheureuse lorsqu'il s'avise de revendiquer la suzeraineté d'une île ou d'une contrée : à l'évidence, Poséidon n'est pas un dieu civique, et sa réussite en ce domaine est des plus improbables ${ }^{13}$. Tout ceci est bel et bon, mais n'explique pas pourquoi, pendant si longtemps,

10 J.G. GRIFFITHS, Atlantis and Egypt, in Historia, 34 (1985), p. 3-28.

11 BRISSON, op. cit. (n. 1), p. 435, y voit une «tare profonde » responsable de « la déchéance de l'Atlantide vers la difformité ».

12 Sur la $\sigma \tau \alpha \dot{\sigma} \sigma i \varsigma$, ce "processus qui fatalement divise la cité en deux », voir N. LORAux, Solon au milieu de la lice, in Aux onigines de l'bellénisme. La Crète et la Grèce. Hommage à Henri Van Effenterre, Paris, 1984, p. 199-214, ainsi que, du même auteur, Thucydide et la sédition dans les mots, in $Q S, 23$ (1986), p. 95-134, et plus particulièrement la page 111.

13 Cf. L. SÉCHAN et P. LÉVÊQUE, Les grandes divinités de la Grèce, Paris, 1966, p. 102. 
Poséidon n'a pas failli à son rôle de divinité poliade ${ }^{14}$. Tout ceci n'explique pas pourquoi, pendant si longtemps, le lien étroit qui unit l'Atlantide à la mer $^{15}$ a pu aller de pair avec un gouvernement juste. On ne pourra, je crois, répondre à ces questions qu'en acceptant l'idée que la chute n'était pas inéluctable, et qu'une cité, pour être maritime, n'est pas automatiquement vouée à la destruction.

\section{Les libations sanglantes}

Dans le rituel sacramentaire, tel qu'il nous est décrit dans le Critias, se rencontrent deux conduites habituellement distinctes dans un sacrifice de serment. Louis Gernet a raison de le souligner : « les rois de l'Atlantide boivent du liquide qui, d'autre part, sert à la libation ${ }^{16}$.

Première conduite : la consommation de vin, pur ou mêlé d'eau, auquel est ajouté le sang de la victime. Liant les contractants les uns aux autres, elle a, selon l'expression de Gernet, un « effet de communion ». Et cela d'autant plus, notons-le, que chacun des participants au rituel s'engage personnellement au moyen d'un caillot de sang qu'il jette dans le cratère. Je crois en effet avec H. Herter que, sur ce point, la traduction d'A. Rivaud doit être corrigée ${ }^{17}$. On ne doit pas lire : « ils remplissaient de sang un cratère et aspergeaient d'un caillot

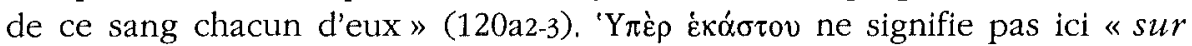
chacun des rois", mais "quant à chacun des rois", "en ce qui concerne chacun des rois», un tel emploi de la préposition étant bien attesté, entre autres chez Platon ${ }^{18}$. Je proposerai donc la traduction suivante : «quant à chacun des rois, il lançait un caillot de sang dans un cratère contenant déjà du vin coupé ${ }^{19}$. L'« effet de communion » est, de surcroît, renforcé par la

14 Poséidon n'est d'ailleurs pas seulement une divinité poliade. Il est aussi, au même titre qu'Athêna, une divinité œciste. Sur ce point, cf. M.-L. DESCLOS, L'Atlantide: une ile comme un corps. Histoire d'une transgression, à parâtre dans les Actes du Colloque Impressions d'îles, Grenoble, 13-15 mai 1992, Toulouse, 1995.

15 L'Atlantide est plus qu'une île : c'est une succession d'îles (Critias, 113d); elle est l'apanage de Poséidon; c'est une puissance maritime; et les métaux dont elle est riche ne sont eux-mêmes, comme l'a montré P. VIDAL-NAQUET, qu'une variété de l'eau (Athènes et l'Atlantide, p. 352-353).

16 L. GERNET, Antbropologie de la Grèce antique, Paris, $1976^{2}$ [Paris 1968], p. 209. Voir l'ensemble du paragraphe consacré au «sacrifice des rois de l'Atlantide », p. 207-217.

17 HERTER, ait. cit. (n. 3), p. 246.

18 Il suffira d'en donner un exemple, que nous fournit le livre III de la République en 387c3-6 :

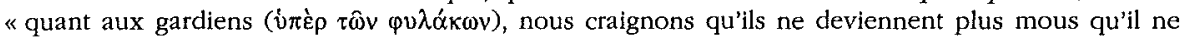
convient ». Cf. J. HUMBERT, Syntaxe grecque, Paris, $1986^{3}$ [Paris, 1945], p. 322, $\$ 555-556$.

19 L. BRISSON, quant à lui, traduit : "ils jetaient au nom de chacun d'eux un caillot de sang ", préservant ainsi ce que je crois être l'essentiel, c'est-à-dire l'engagement personnel de cbacun dans la préparation du breuvage devant servir aux libations après avoir été rituellement consommé. 
nature du breuvage : «le sang de la victime», pur ou coupé, «par contact ou par absorption » servant «à créer un engagement réciproque ${ }^{20}$.

Deuxième conduite: la libation accompagnant le sacrifice de serment, en se rappelant que le liquide répandu est celui qui va être consommé. Il se peut, comme P. Stengel en fait l'hypothèse, que le sang et le vin aient un pouvoir négatif, c'est-à-dire suscitent la poursuite et la punition des parjures par des puissances vengeresses ${ }^{21}$. Dans ce cas on serait fondé, avec A. Vincent, à voir dans le sacrifice des rois atlantes « un sacrifice de communion en vue d'une ordalie ${ }^{22}$. Mais ils peuvent également avoir un pouvoir positif, comme le rappelle $H$. Herter : le breuvage peut fortifier ceux qui le consomment, il peut avoir une efficacité «cathartico-apotropaïque ${ }^{23}$. Cependant, et en tout état de cause, quelle que soit l'interprétation retenue, si tant est qu'il faille en choisir une, par la libation un pacte est scellé entre ceux qui l'accomplissent, visant à préserver la communauté de ce qui pourrait, de l'intérieur, venir la menacer ${ }^{24}$. Nous sommes également en présence d'un serment promissoire en ce qu'il porte sur le futur proche ou lointain, les rois, on s'en souvient, s'engageant mutuellement à « rendre la justice en fonction des lois inscrites sur la stèle, (à) châtier celui d'entre eux qui, antérieurement, y aurait contrevenu sur quelque point, (à) ne contrevenir de leur plein gré à l'avenir sur aucun point aux ordres de la prescription, (à) ne commander et (à) n'obéir que conformément aux lois de leur père » (Critias, 120a5-b2) ${ }^{25}$. Cette fois le lien est établi entre les rois atlantes et le dieu-père qu'ils ont invoqué. On est donc en présence d'un rituel qui a pour but et pour effet de resserrer périodiquement les liens : de façon borizontale, entre ceux qui exercent le pouvoir; de façon verticale, entre les rejetons du dieu et leur père divin. Notons que, dans ce dernier cas encore, il s'agit d'assurer «la cohésion de la communauté civique et le bon fonctionnement des institutions qui en conditionnent l'activité ${ }^{26}$. En témoigne le contenu des lois par rapport auxquelles les rois vont avoir à juger et à être jugés : elles concernent toutes le commander et l'être-commandé ${ }^{27}$. Et toutes

20 GERNET, op. cit. (n. 16), p. 209.

21 P. STENGEL, Die griechischen Kultusaltertümer; München, 1920, p. 123.

22 VINCENT, art. cit. (n. 5), p. 90.

23 HERTER, art. cit. (n. 3), p. 252.

24 Cf. supra note 2. Voir également J. RUDHARDT, Notions fondamentales de la pensée religieuse et actes constitutifs du culte dans la Grèce classique, Paris, 1992 2 [Paris 1958], p. 245.

25 L'efficace du serment promissoire est d'autant plus grande que Poséidon est à la fois garant du respect de la parole donnée, juge des actions futures, et auteur des institutions légales de la grande île.

26 RUDHARDT, op. ctt. (n. 24), p. 207.

27 À savoir : - l'hégémonie de l'aîné du premier couple de jumeaux et de sa descendance; - l'autorité de chacun des dix sur le territoire qui lui revient et sur les citoyens de sa cité; - l'interdiction de prendre les armes les uns contre les autres; - l'obligation de s'entre-secourir si l'un d'entre eux avait tenté de chasser l'une des races royales; - l'impossibilité de mettre à mort 
visent à éloigner le péril de la guerre civile, en sorte que la division stasiôtique ne vienne pas se substituer à une division du pouvoir d'où, pourtant, la hiérarchie n'est pas absente : l'hégémonie ira à l'aîné du premier couple de jumeau et à sa descendance ${ }^{28}$. Que cette hégémonie d'Atlas et de sa lignée sur les autres rois et leur descendance, comme d'ailleurs celle de chaque roi sur les citoyens de sa cité, puisse dégénérer en tyrannie, la chose est possible et a d'ailleurs déjà été soulignée ${ }^{29}$. De surcroît, tout dans le texte nous incite à voir dans cette dégradation l'origine politique du sort funeste de la grande île : le contenu des lois gravées sur la colonne, et le péril qu'elles ont pour fonction d'éloigner; la première désobéissance, de la part de ceux de la race d'Atlas, à la volonté du dieu fondateur ${ }^{30}$; la référence enfin à la dodécarchie égyptienne, et aux circonstances de la prise du pouvoir par Psammétique. Mais que la chose fût possible, et qu'elle n'ait pas été évitée, ne la rendait pas pour autant inévitable. Telle est sans doute la plus grande différence entre le récit platonicien et le récit hérodotéen auquel on l'a comparé.

\section{La prise du pouvoir par Psammétique}

Hérodote, dans le livre II des Histoires, nous décrit la situation politique de l'Egypte avant que Psammétique ne s'empare du pouvoir. Le pays, partagé en douze lots, est gouverné par douze rois. Ces rois, nous dit Hérodote, "s'unirent par des mariages et régnèrent en se faisant une loi de ne pas s'entre-détruire, de ne pas chercher à avoir plus l'un que l'autre, et d'être étroitement unis » (II, $147,8-11)$. Cette loi trouve son $\alpha$ it tov dans un oracle prédisant que « celui d'entre eux qui ferait des libations avec une coupe de bronze dans le sanctuaire d'Héphaistos règnerait sur l'Egypte entière ». Tout se passe pour le mieux jusqu'au jour où :

l'un des dix rois sans l'accord de la majorité; - l'obligation de délibérer en commun au sujet de la guerre et des autres affaires.

28 «Il l'établit roi $(\beta \alpha \sigma \imath \lambda \varepsilon ́ \alpha)$ sur tous les autres ( $\tau \hat{\omega} v \not \alpha \lambda \lambda \omega v)$; tandis que ces autres il les instituait seulement gouverneurs (ă $\rho \chi 0 \vee \tau \alpha \varsigma) »(C r t t i a s, 114 \mathrm{a} 2-3$; je ne suis pas la traduction de L. Brisson). GRIFFITHS, art. cit. (n. 10), p. 19, 27) identifie ce pouvoir d'un $\beta \alpha \sigma i \lambda \varepsilon v ́ \varsigma$ sur des

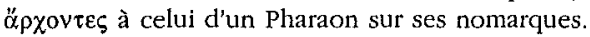

29 Cf. VIDAL-NAQUET, op. cit. (n. 6), p. 355-356: «Dans sa circonscription, chaque roi est un souverain absolu, jouissant du droit de vie et de mort, ce qui peut correspondre aussi bien, s'il s'agit d'un philosophe, au statut du Politique idéal, que dans le cas contraire, à la tyrannie ». La forme même de la cité-capitale primitive se prête à cette double lecture, puisqu'elle peut tout aussi bien être considérée comme une imitation réussie de ce Vivant parfait qu'est le Monde (cf. Tim., 33b), que comme une rêplique de la cité du tyran, dressée sur un pic et ceinte de murailles (Théét., $174 \mathrm{e})$.

30 Ce sont les descendants d'Atlas qui jettent des ponts sur les bras de mer (115c), et ouvrent des passages dans les enceintes de terre (115d), rompant ainsi l'isolement de l'île centrale, dont

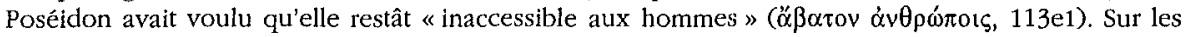
conséquences d'une telle transgression, cf. DEsclos, art. cit. (n. 14). 
comme ils offraient un sacrifice dans le sanctuaire d'Héphaistos et que, le dernier jour de la fête, ils allaient faire des libations, le grand prêtre leur apporta des coupes d'or dont ils se servaient habituellement pour cela; mais il se trompa sur le nombre et en apporta onze alors qu'ils étaient douze. N'ayant pas de coupe, celui d'entre eux qui se tenait le dernier, Psammétique, ôta son casque, lequel était de bronze, le tendit et fit les libations avec. Tous les autres rois, eux aussi, portaient des casques et se trouvaient les avoir sur la tête. Ce fut donc sans nulle pensée perfide que Psammétique tendit le sien. Mais les autres rois rapprochèrent dans leur esprit ce qu'il avait fait et l'oracle qui leur avait été rendu, à savoir que celui d'entre eux qui ferait des libations avec une coupe de bronze, celui-là serait seul roi de l'Égypte (II, 151, 1-13).

Psammétique est immédiatement exilé et dépouillé de sa puissance, mais aidé par des Ioniens et des Cariens, il finit par renverser les autres rois et par s'emparer du pouvoir.

À lire ces deux textes ensemble, on comprend que l'on ait pu voir, dans ce passage des Histoires, l'une des suites possibles de ce dialogue inachevé qu'est le Critias $^{31}$. L'Atlantide, minée par des luttes intestines, sombrerait sous les coups de l'Athènes primitive ${ }^{32}$. Mais on comprend moins comment, après être apparue «comme un de ces mixtes réussis que définissent le Politique, le Timée, le Pbilèbe et les Lois », elle a pu se laisser "gagner par la désunion et la dissemblance ${ }^{33}$. Sauf à considérer, ce qui fut fait, que cette issue était, dès

31 Gill, op. cit. (n. 9), p. 69, note à Critias, 119d7sq. Sur la question de l'inachèvement du Critias, il faut signaler par ailleurs l'article d'A. LuKINOVICH, Un fragment platonicien: le «Critias", in La question du fragment, séminaire interdisciplinaire, semestre d'été 1981, sous la direction des professeurs J. BOUVERESSE, L. DÄLLENBACH, A. HURST, Publication de la Faculté des Lettres de l'Université de Genève, p. 43-47, qui voit dans le caractère fragmentaire du Critias une façon de signifier le continuel inachèvement d'un discours philosophique écartelé entre son désir de vérité et les limites de la connaissance discursive. Un second article, de M. LAPLACE, Le "Critias" de Platon, ou l'ellipse d'une épopée, in Hermes, 112 (1984), p. 377-382, considère que "le châtiment infligé par Zeus à l'Atlantide a déjà été raconté par Homère ». Le lecteur de Platon trouvera donc la suite du Critias dans l'Iliade.

32 Ce sont ces luttes intestines, conséquences de l'« injuste cupidité » et des « excès » atlantes, qui expliqueraient l'intervention de Poséidon (« le dieu (...) l'assembla pour la diriger contre nos contrées », Critias, 120d7-8) sans pour autant que cette intervention contredise à la bonté des dieux affirmée au début du dialogue. La « dépravation » de cette race auparavant excellente nécessite la manifestation de la justice divine. Que cette manifestation passe par l'incitation à une faute autre, porteuse d'un châtiment qui articule l'action des hommes et le vouloir des dieux, n'a rien qui doive « surprendre », contrairement à ce que semble penser L. BRISSON (op. cit. [n. 1], p. 392 n. 189). Elle est en tout point semblable à l'épisode du songe divin qui pousse Xerxès, contre sa volonté première, à envahir la Grèce et à marcher ainsi à la rencontre de son destin (Hérodote, VII, 12-18). Cf. C. DARBO-PESCHANSKI, Thucydide: bistorien, juge, in Mètis, 2 (1988), p. 109-140, qui montre comment la campagne contre la Grèce est à la fois un nouvel acte de démesure et l'instrument grâce auquel seront enfin châtiées la violation, constante depuis Crésus, des frontières géographiques et, à travers elle, la négation du «partage fondamental entre les différentes régions de la terre $\gg(\mathrm{p} .114)$.

33 VIDAL-NAQUET, op. cit. (n. 6), p. 356, 360. 
l'origine, inscrite dans la structure même de la grande île ${ }^{34}$. Semble militer en faveur de cette lecture le constat, incontestable, selon lequel le récit de Critias est, d'entrée de jeu, placé sous le signe de la otóoıc. D'entrée de jeu, c'est-àdire, d'abord, dès le proème du Timée, lorsque Socrate rattache nos deux dialogues à ces discours «qui furent tenus hier» sur la constitution la meilleure, et sur les citoyens qui doivent la mettre en œuvre. Par la communauté des biens, des femmes et des enfants, la République, en effet, pense la cité sur le modèle de la famille. Plus : transforme la cité en une immense famille d'où sera absent le plus grand mal, celui qui la divise et d'une en fait plusieurs (Rép., V, 462a9-b1). D'entrée de jeu, c'est-à-dire, aussi, dès l'annonce par Critias du récit qui lui fut conté par son grand-père lorsqu'il était encore enfant : récit solonien inachevé, du moins dans sa mise en forme, pour cause de séditions ( $\delta$ ì̀ $\tau \grave{\alpha} \varsigma \sigma \tau \alpha \dot{\alpha} \sigma \varepsilon ı \varsigma, T i m ., 21 c 6-7$ ). Il n'est pas jusqu'aux dernières lignes de notre dialogue qui ne nous mettent en présence d'un processus semblable à celui que décrit Thucydide (III, 82, 4) lorsque la guerre

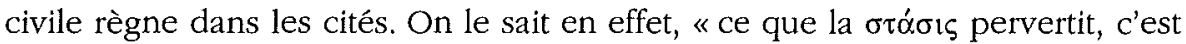
avant tout le maniement de l'éloge et du blâme »35. Or c'est bien à une telle perversion des mots et des esprits que l'on assiste lorsque passent «pour être

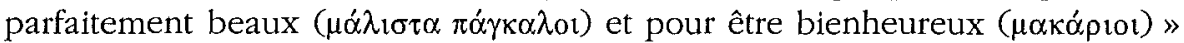
ceux qui, tout à l'inverse, sont emplis « d'injuste cupidité et d'excès

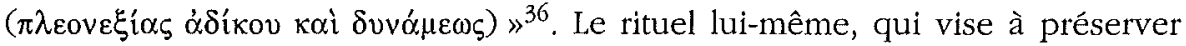
l'harmonie et à rendre impossible tout conflit entre les dix rois, «laisse planer un doute sur la constance de ces rapports harmonieux $\gg^{37}$.

Néanmoins, ceux-là mêmes qui voient dans le «triomphe total de l'altérité » le «destin de l'Atlantide», ou qui font de sa «déchéance vers la difformité » un « inévitable ${ }^{38}$ ne manquent pas de rappeler l'étrange ambivalence d'un système politique qui peut tout aussi bien s'apparenter à l'exercice du pouvoir par le gouvernant idéal qu'à celui du tyran ${ }^{39}$. Ils s'arrêtent sur la signification des noms des premiers rois, noms qui mettent en évidence leurs qualités, tant physiques que morales, et leur aptitude à gouverner. Ils soulignent la réussite, marquée au coin de la durée ${ }^{40}$, des institutions légales données par

34 VIDAL-NAQUET, op. cit. (n. 6), p. 353; p. 352-357, l'auteur relève tous les traits qui font de l'Atlantide, selon sa propre expression, le «monde de la duplication».

35 N. LORAuX, Thucydide et la sédition dans les mots, art. cit. (n. 12), p. 104. Il convient de

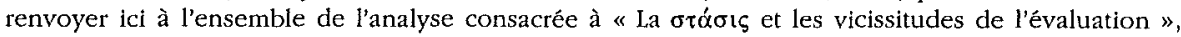
p. 102-109.

36 Critias, 121b5-7. Voir également, pour une semblable perversion du " maniement de l'éloge

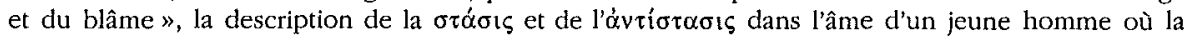
faction démocratique est en lutte contre la faction oligarchique (Rép., VIII, 560d3-6 et 560e5-561a1).

37 BRISSON, op.cit. (n. 1), p. 435.

38 VIDAL-NAQUET, op. cit. (n. 6), p. 351 et 360; BRISSON, op. cit. (n. 1), p. 435.

39 Cf. supra n. 29.

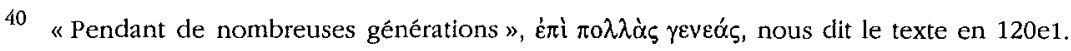


le dieu à ses enfants et à leur postérité, et renforcées, selon le mot de Luc Brisson, par la conjonction « d'un réseau mathématique important et du cérémonial ${ }^{41}$. Et pourtant, en ce temps-là aussi, l'Atlantide était une île, et sa structure portait déjà le sceau de l'altérité. Dès lors, comment expliquer cette "évolution désastreuse ${ }^{42}$ ? Luc Brisson comme Pierre Vidal-Naquet s'accordent pour reconnaître le « juste équilibre » du régime politique atlante, équilibre rendu «précaire », cependant, à la fois par la double origine mortelle et divine - des gouvernants, et par l'influence délétère des richesses à leur disposition. Lorsque l'élément humain vient à dominer en eux, les rois se révèlent impuissants à lutter contre cette influence ${ }^{43}$. Soit. Je crois cependant qu'il faut se garder ici de confondre cause première et causes auxiliaires.

Avec le récit de Critias, et il en est de même pour le discours de Timée, nous entrons dans le monde de ce qui est mû, c'est-à-dire dans le monde du devenir. Les propos de Socrate sont, sur ce point, sans ambiguïté ${ }^{44}$. L'Atlantide, il ne faut jamais l'oublier, est donc, comme l'Athènes primitive, une chose en devenir vouée en tant que telle à la destruction; en tant que telle, et non en raison de sa structure, du naturel de ses dirigeants, ou de la nature de sa constitution. L'Athènes primitive est terrienne, nous dit-on, et s'il faut en croire la cosmologie du Timée (56d), « des quatre éléments la terre est précisément celui qui ne peut se transformer ${ }^{45}$. Elle n'échappera pourtant pas à la destruction. Sa «structure » n'est donc pas en cause, qui dit, tout au contraire, la stabilité et la permanence. Il n'y a, par conséquent, aucune raison de voir dans le caractère maritime de l'Atlantide la cause d'une destruction dont on ne rend pas le caractère terrien d'Athènes responsable. Tout au plus les dirigeants doivent-ils, en raison même de ce caractère maritime, faire preuve d'une plus grande vigilance dans le respect et l'application de lois justes et bonnes. Par

41 Sur l'ensemble de ces points, cf. VIDAL-NAQUET, op. cit. (n. 6), p. 355-356; BRISSON, op. cit. (n. 1), p. 422-424, 429-430, 435-436.

42 VIDAL-NAQUET, op. cit. (n. 6), p. 359.

43 VIDAL-NAQUET, op. cit. (n. 6), p. 359; BRISSON, op. cit.(n. 1), p. 435. Il faut peut-être voir là une confirmation de ce qu'affirme la République à propos des prescriptions relatives à l'accouplement des animaux de race : "Et si l'on ne donne pas ces soins à la génération, tu penses bien que la race de tes oiseaux et de tes chiens dégénérerait considérablement. Et pour les autres vivants

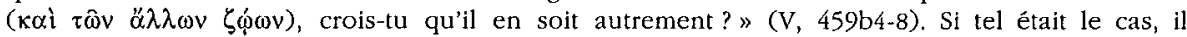

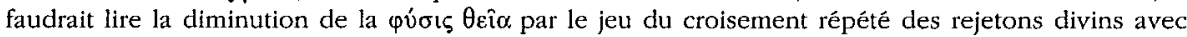
des femmes mortelles, certes comme un inéluctable biologique, mais aussi comme l'indice et l'effet d'une carence païdeutique. Car, il ne faut pas l'oublier, de telles prescriptions rentrent dans le cadre plus général de la $\tau \rho \circ \varphi^{\prime}$ et de la $\pi \alpha 1 \delta \varepsilon i ́ \alpha$ qu'il convient de dispenser à tous les vivants, tant il

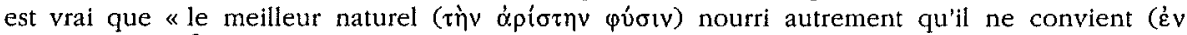

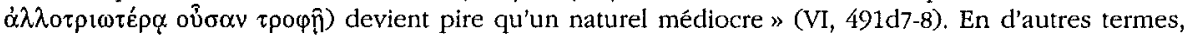
il en serait des mâles royaux de l'île Atlantide comme des chiens, des oiseaux ou des chevaux de belle race: on ne doit pas s'en remettre au hasard pour les accouplements et la procréation, mais s'efforcer d'aparier les naturels autant qu'il est possible. $C f$. infra, section 4 : « Nécessité d'une $\pi \alpha 1 \delta \varepsilon i \alpha$ des princes ».

44 Tim., $19 \mathrm{~b} 8$.

45 VIDAL-NAQUET, op. cit. (n. 6), p. 348. 
ailleurs, l'onomatothèse du Critias (114b-c), tout comme le jugement porté, à la fin de ce dialogue, sur les qualités morales des dix rois (120e-121a), disent assez leur bon naturel. Quant à la constitution de la grande île, elle est due à un nomothète divin, et l'on sait par la lecture des Lois (I, 624a-625a) que c'est là gage d'excellence. Je ne dirai rien de la gémellité royale, en quoi l'Athénien reconnaît la prévoyante prudence d'un dieu ${ }^{46}$; rien non plus de la primauté accordée à l'aîné des deux plus vieux, mêlant ainsi « la puissance raisonnable de la vieillesse à la force présomptueuse de la race ${ }^{47}$; rien enfin de «l'union d'une nature humaine à une nature divine » qui fait le bon législateur ${ }^{48}$. De même, on a voulu voir, dans l'infinie variété de la faune et de la flore de l'île d'Atlas, une représentation des «progrès de l'altérité qui triomphe dans la

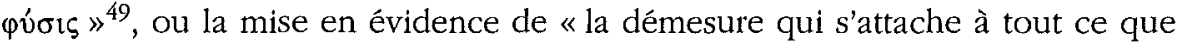
recèle l'Atlantide ${ }^{50}$. Mais on peut tout aussi bien interpréter cette abondance comme une imitation, là encore réussie, de ce Vivant qu'est le Monde, lequel doit comprendre en lui tous les vivants ( $\tau \dot{\alpha} \pi \alpha \dot{v} v \tau \alpha \zeta \hat{\omega} \alpha$ ) pour " ressembl[er] le plus possible au vivant parfait et intelligible » qui lui sert de modèle ${ }^{51}$. Ce que semble confirmer la forme de la capitale atlante, dont la figure circulaire est doublement parfaite: parce qu'elle est «celle où s'inscrivent toutes les autres figures », et parce que, ce faisant, elle est l'image du Monde, «qui doit envelopper en lui-même tous les vivants ${ }^{52}$. Ce que semble également confirmer, me semble-t-il, la similitude du geste divin, et des mots pour le dire, dans nos deux dialogues : le Poséidon du Critias façonne la cité au tour en partant du centre de l'île ${ }^{53}$, à la façon du Démiurge du Timée qui « a tourné le monde en forme sphérique et circulaire (...) depuis le centre jusqu'aux extrémités ». Il faut conclure : si l'Atlantide est détruite et entre en déchéance, ce n'est pas en raison de sa nature, de celle de ses rois ou de celle de ses institutions légales ${ }^{54}$.

46 «Un dieu qui prenait soin de vous et qui, en prévision de l'avenir, faisant naître chez vous deux rois jumeaux au lieu d'un seul, a ramené l'autorité à des limites plus justes » (Lois, III, 691d8e1).

47 Lois, III, 692a1.

48 Lols, III, 691e1-2. Éd. DES PLACES, n. 2 p. 33 de son édition des Lois dans la C.U.F., reconnaît Lycurgue dans cette description.

49 VIDAL-NAQUET, op. cit. (n. 6), p. 354.

50 BRISSON, op. cit. (n. 1), p. 425.

51 Tim., 39e. Voir également 92c6-10.

$52 T i m ., 33 \mathrm{~b}$.

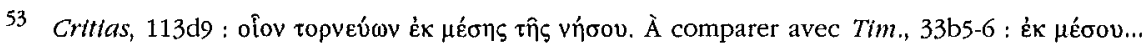

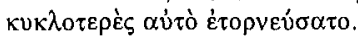

54 Ce qui ne signifie pas que, pour Platon, cité maritime et cité terrienne aient même valeur, que la cité maritime ne soit pas plus aisément victime des germes de désintégration qu'elle porte en elle, et qu'il ne soit pas politiquement préférable, lorsque cela est possible, de fonder un état nouveau loin de la mer plutôt qu'à sa proximité. Les développements des livres IV (704a-b) et $\mathrm{V}(745 \mathrm{~b}-\mathrm{e})$ des Lois suffiraient, s'il en était besoin, à prouver le contraire. Cela signifie encore moins que je remets en question la pertinence des analyses qui ont lu l'bistoire et la géograpbie 
C'est tout simplement parce que « tout ce qui naît est sujet à la corruption » (Rép., VIII, 546a2), et cela est vrai, aussi, de la Kallipolis.

La comparaison avec le livre II des Histoires est, en ce domaine, riche d'enseignement. À trop s'arrêter sur ce qui rapproche les deux textes, on a fini par passer sous silence leur différence fondamentale. Alors que la fin de la dodécarchie égyptienne, et sa cause, sont d'entrée de jeu annoncées par un oracle, il n'y a rien de tel en ce qui concerne l'Atlantide. Ainsi, tandis que l'oracle constitue "le ressort narratif essentiel " ${ }^{55}$ de ce passage des Histoires, il est totalement absent du récit platonicien. Dans le premier cas, les lois édictées par les douze souverains n'ont d'autre but que de retarder l'inéluctable, révélé par le dieu. Dans le second cas, elles émanent directement de Poséidon, et doivent permettre à des gouvernants d'origine divine de régner dans le respect de la justice. C'est leur transgression qui vaudra châtiment à ceux de la race d'Atlas, tandis que Psammétique agit "sans nulle pensée perfide » ${ }^{56}$. C'est d'ailleurs pourquoi les autres rois se refusent à le mettre à mort : «se

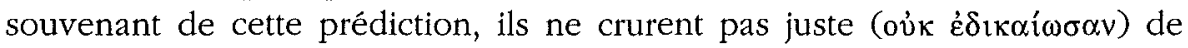
mettre Psammétique à mort, parce qu'en le questionnant ils constatèrent qu'il

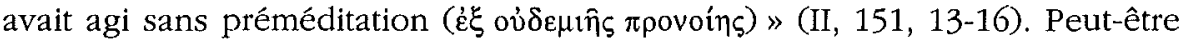
le lecteur moderne s'étonnera-t-il de la «négligence», du manque de «lucidité », de l'« insouciance » des onze rois. Pour Hérodote en tout cas, leur attitude est la seule qui vaille, précisément parce qu'il n'y a pas faute de la part de Psammétique. Ainsi s'explique qu'ils « ne prennent aucune mesure pour neutraliser définitivement leur collègue ${ }^{57}$ : il y aurait déséquilibre entre l'offense et la réparation, ce qui scellerait leur perte ${ }^{58}$. Face à l'acte de Psammétique, ils n'ont par conséquent aucune marge de manœuvre: quoi qu'ils fassent, quoi qu'ils décident, ce qui doit être sera. L'avenir des Atlantes, en revanche, est entre leurs mains, et leur défaite devant les Athéniens est le prix à payer pour leur démesure.

Mais, pour que la justice divine ne soit pas un vain mot, et que la fable platonicienne ait une quelconque efficacité politique, encore fallait-il que la faute pût ne pas être commise, qu'elle ne fût pas inscrite dans la nature de la grande île.

\footnotetext{
atlantes comme autant de manifestations du «déploiement », du «triomphe » même, « de l'apeiron, de l'altérité » VIDAL-NAQUET, op. cit. [n. 6], p. 353-354). Je voudrais simplement montrer que ce " triomphe » et ce « déploiement » 1) sont pensables, mais peuvent être pensés autrement; 2) sont des possibles, certes réalisés, mais qui demeurent contingents.

55 C. DARBO-PESCHANSKI, Le discours du particulier. Essat sur l'enquête bérodotéenne, Paris, 1987, p. 74.

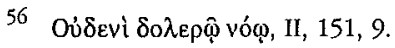

57 DARBO-PESCHANSKI, op. cit. (n. 55), p. 75.

58 DARBO-PESCHANSKI, op. cit. (n. 55), p. 43-74
} 


\section{Nécessité d'une $\pi \alpha 1 \delta \varepsilon i ́ \alpha$ des princes}

Je le rappelais au début de cet article, l'éducation apparaît comme un critère déterminant dans le choix des orateurs qui doivent donner vie à la Cité idéale dépeinte dans la République, dialogue dont les pages les plus fortes sont consacrées à établir avec précision quel type de $\pi \alpha 1 \delta$ cí $\alpha$ il convient de dispenser à ceux qui auront pour tâche d'en être les gardiens. L'importance de l'activité païdeutique est réaffirmée avec force dans le Timée et le Critias, puisque ce sont les dieux qui, après le lotissement fait par Dikê des différentes

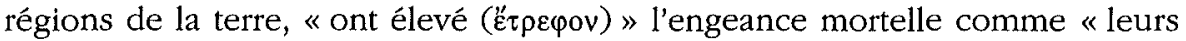
possessions et leurs propres troupeaux »(Critias, 109c1) $)^{59}$. Conformément à cette règle générale, c'est Athéna " qui a élevé et instruit (e̋ $\theta \rho \varepsilon \psi \varepsilon v$ к $\alpha$ ì غ̇ $\pi \alpha \hat{i} \delta \varepsilon v \sigma \varepsilon v$ ) $\gg$ la cité d'Athènes (Tim., 23d7). Puis, relayant l'action de la déesse, ce fut à des hommes divins d'isoler le groupe des combattants des autres

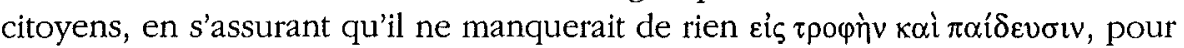
son instruction et son éducation (Critias, 110c7). Où l'on retrouve les enseignements de la République, brièvement rappelés par Socrate au début du Timée, en ce qui concerne l'éducation ( $\tau$ popи́) des gardiens : « La gymnastique, la musique, ainsi que les sciences toutes autant qu'elles conviennent à leur condition, n'est-ce pas en tout cela qu'il les faut élever $\left(\tau \varepsilon \theta \rho \alpha \hat{\alpha} \varphi \theta \alpha_{l}\right)$ ? $»^{60}$. À procéder ainsi on s'assurera en effet que ceux qui auront été élevés ( $\tau \rho \alpha \varphi e^{v} \tau \tau \varsigma$ ) de la sorte n'auront plus que dédain pour les richesses, faisant de la vertu leur unique souci ${ }^{61}$. À l'inverse, c'est par l'effet «d'une éducation mal

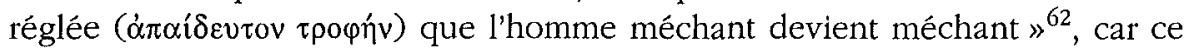
n'est que grâce à l'éducation ( $\delta i \grave{\alpha} \tau p o \varphi \hat{n} \varsigma$ ) que l'on peut fuir le vice et choisir son contraire ${ }^{63}$. Poséidon ne faillira pas à sa tâche de divinité poliade : «il

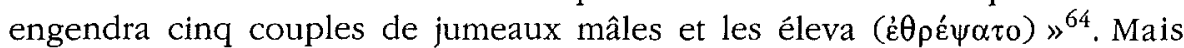
alors qu'à Athènes des hommes divins prolongèrent l'action de la déesse, rien de tel ne se produisit chez les Atlantes. Dans la minutieuse description qui occupe une grande partie du Critias (113b7-121c3), il ne sera pas fait une seule

59 Notons qu'après cette déclaration suivent les noms d'Athéna et d'Héphaistos qui, en raison de leur double amour de la sagesse et de l'art, " après avoir rendu gens de bien les hommes issus

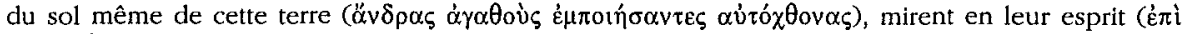

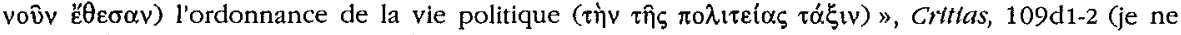
suis pas la traduction de $\mathrm{L}$. Brisson).

60 Tim., 18a10-11. Gf. Rép., II, 376e sq; III, 403c sq; 410c sq.

61 Tim., 18b1-8. Cf. Rép., III, 416d-417b; V, 464b-c sq.

62 Tim., 86e2. Voir également Lots, VI, 766a : «L'homme est sans doute, nous l'affirmons, un être apprivoisé; néanmoins, si avec une bonne éducation et un naturel heureux il devient d'ordinaire le plus divin et le plus doux de tous les êtres, faute d'une éducation suffisante et bien conduite c'est le plus sauvage de tous ceux que la terre produit ».

63

Tim., $87 \mathrm{~b} 7$.

64

Critias, $113 \mathrm{e} 7$. 
fois mention d'une quelconque activité éducative. De longues pages, en revanche, sont consacrées à l'aménagement de l'espace (115b7-118e7). La chose, je crois, n'est pas indifférente. On s'en persuadera si l'on compare le Critias au texte dont il est le prolongement. Ainsi, lorsque, au livre IV de la République en 424a5, Socrate affirme «qu'une cité qui a bien commencé va

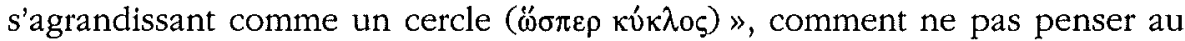
geste du dieu bâtisseur lorsqu'il aménage la hauteur qu'il occupe avec la mortelle Clitô? L'isolant en cercle ( $\kappa$ $\kappa \lambda \hat{\varphi})$, il l'entoura d'anneaux alternés de terre et d'eau "de tous côtés équidistants du centre de l'île, comme s'il eût fait marcher un tour de potier " ${ }^{65}$. Mais, de la République au Critias, le «cercle» a changé de nature. Là où Socrate entreprend de former au tour la cité par

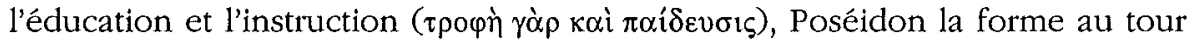
par l'urbanisme. Mais il sait aussi, on l'a vu, se faire éducateur. Il n'en sera pas de même pour ses descendants, qui se contenteront de construire, ou d'embellir les constructions précédentes. Or l'aménagement de l'espace doit aller de pair avec «l'aménagement des âmes », faute de quoi les suivants ne deviennent pas meilleurs, mais pires que leurs devanciers ${ }^{66}$. Tel est, je crois, l'enseignement qu'il faut tirer de la lecture conjointe de ces deux passages.

Certes, tant que perdureront les effets de la $\tau \rho \circ \varphi$ divine, les rois, conformément aux affirmations du Timée et de la République, "dédaignant toutes choses à l'exception de la vertu », feront «peu de cas de leur prospérité », regardant comme un «fardeau la masse de leur or et de leurs autres biens » ${ }^{67}$. «Pendant de nombreuses générations", nous dit le Critias, « et tant que la nature du dieu domina en eux, les rois restèrent dociles à la voix de leurs lois

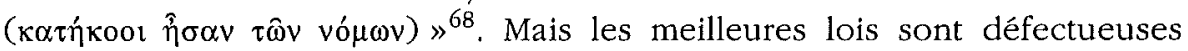
$(\varphi \alpha \hat{v} \lambda \alpha)$, et les meilleurs naturels s'altèrent et dégénèrent, si l'instruction et

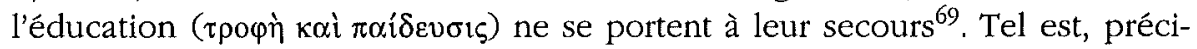
sément, ce qui est advenu à la descendance des cinq premiers couples de jumeaux royaux, «Zeus, qui règne en s'appuyant sur des lois ${ }^{70}$ ne $s^{\prime} y$ est

65 Critias, $113 \mathrm{~d}$.

66 Cet «aménagement des âmes » est assuré par une $\tau \rho \circ \varphi \eta ́$ et une $\pi \alpha 1 \delta \varepsilon i ́ \alpha$ appropriées. Il est irréalisable lorsqu'elles font défaut, ou lorsqu'elles sont corrompues. Il faut peut-être voir ici une critique du remodelage clisthénien de l'espace civique, lequel va de pair avec l'apparition de valeurs démocratiques qui évincent les anciennes valeurs aristocratiques. $C f$. P. LÉVÊQUE et P. VIDAL-NAQUET, Clisthène l'Atbénien. Essai sur la représentation de l'espace et du temps dans la pensée politique grecque de la fin du Vle siècle à la mort de Platon, Paris, 1964, particulièrement les chapitres I, II et VIII; Cl. MOssÉ, Histolre d'une démocratie: Athènes, Des origines à la conquête macédonienne, Paris, 1971, p. 25-30.

67 Critias, $120 \mathrm{e} 6-121 \mathrm{a} 2$.

68 Critias, $120 \mathrm{e} 1-2$.

69 Rép., IV, 423e. Cf. supra n. 42. Le naturel philosophe lui-même n'échappe pas à la règle : $c f$. Rép., VI, 495a-b.

70 Critias, 121 b7. 
pas trompé qui «voulut leur appliquer un châtiment, afin de les faire réfléchir et de les ramener à plus de modération » ${ }^{71}$.

Voilà néanmoins qui sonne étrangement aux oreilles de celui qui se souvient encore du premier récit de Critias : "l'île Atlantide s'abîma dans la mer et disparut $~^{72}$. Il n'y eut point de survivants pour tirer leçon de la défaite. Sauf à considérer, et c'en est sans doute là une preuve supplémentaire, que par-delà ou par-deçà les colonnes d'Hercule ce sont, selon le mot de Bartoli, «des Athéniens toujours, des Athéniens de tous côtés » ${ }^{73}$. Est-ce à dire que la fable platonicienne n'a d'autre but que de raviver le souvenir de la défaite sicilienne et d'amener ainsi les Athéniens «à plus de modération »? Je crois qu'il y a plus : l'affirmation qu'une cité maritime peut être juste et ses gouvernants vertueux. N'est-ce pas la fonction d'Hermocrate, dont Socrate

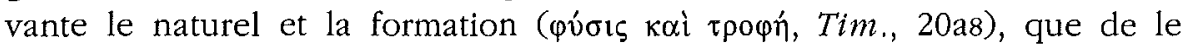
rappeler ? La Sicile, par la seule présence du général syracusain, sert de toile de fond à ce «conte des deux cités », mais ce n'est pas dans le sens où on l'entend habituellement. Cette île n'agresse pas, elle subit l'agression; elle préfère l'union à une guerre intestine (oikzíos $\pi$ ó $\lambda \varepsilon \mu \circ)^{74}$; elle sort victorieuse, enfin, de son affrontement avec l'armée athénienne. Pour le dire d'un mot : elle joue en filigrane le rôle d'une anti-Atlantide ${ }^{75}$. De la même manière, le sort d'Athènes n'est pas scellé par sa situation géographique, et il est encore temps d'opérer cette conversion salutaire qui éloignerait le péril. Mais encore faudraitil, pour cela, modifier l'organisation de l'espace et la formation des âmes. Seul, en effet, un «bon régime d'éducation ${ }^{76}$, peut permettre à l'être humain de retrouver son bon sens lorsqu'il est la proie de ce mouvement ininterrompu de flux et de reflux qui est celui de la nourriture pour le corps, et de la mer pour les cités. Faute d'en être pourvus, les rois atlantes, après avoir fait communiquer le port et l'île centrale, se trouvèrent impuissants à maîtriser et à dominer les effets de ce grand flot sur leur âme ${ }^{77}$. Notre texte est donc doublement

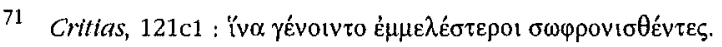

72 Tim., 25d2-3.

73 G. BARTOLI, Essai sur l'explication historique donnée par Platon de sa République et de son Atlantide, en tête des Réflexions impartiales sur le progrès réel ou apparent que les sciences et les arts ont fait dans le XVIIIe siècle en Europe, Paris, 1780, cité par P. VIDAL-NAQUET, Hérodote et l'Atlantide, art. cit. (n. 6), p. 45.

74 THUC, IV, 64, 5. C'est Hermocrate qui parvint à convaincre les Siciliens de faire le choix de l'union (IV, 58-65).

75 Certes, la Sicile a pu, et pourra, être «mal gardée », ou livrée à de mauvais gardiens. Mais cet état de chose n'a rien d'une fatalité, l'insularité ne constituant pas en soi un obstacle infranchissable à la réalisation d'une constitution bonne. Platon l'eût-il cru qu'il n'aurait pas, par trois fois, abordé son rivage.

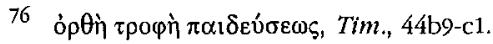

77 Il convient de lire ensemble les lignes consacrées aux effets de la nourriture sur le corps et sur l'âme (Tim., 43a-b), à l'âme de la nourriture et au foie, qui lui sert de base physiologique (Tim., 70e-71a) et à la description du grand port de l'Atlantide (Critias, 117e). Cf. DESCLOS, art. cit. (n. 14). 
païdeutique : en ce qu'il montre concrètement les conséquences d'un défaut d'éducation, ou d'une éducation défectueuse, et en ce qu'il indique le moyen permettant de les éviter ${ }^{78}$. Si le Critias est inachevé, c'est peut-être, aussi, parce qu'il revenait aux Athéniens d'en écrire la fin. Une façon comme une autre de dire que les hommes font l'histoire.

Université de Grenoble 2

Marie-Laurence DEScLos

Département Philosophie

Bât. Sciences de l'Homme

BP $47-1251$ av. Centrale

F - 38040 GRENOBLE Cedex 9

78 En ce sens, le double récit de Critias excède ce que Socrate attendait de lui, puisqu'il ne se contente pas de montrer que la cité idéale, lorsqu'elle entre en guerre, «fait voir les qualités qu'ont transmises aux citoyens leur éducation et leur formation » (Tim., 19c). 